ISSN 1980-5098@ @ @ \& DOI: http://dx.doi.org/10.5902/1980509831652

\title{
INFLUÊNCIA DA TOPOGRAFIA E DA ABERTURA DO DOSSEL NA ESTRUTURA DO COMPONENTE HERBÁCEO-ARBUSTIVO EM DOIS FRAGMENTOS FLORESTAIS NA PLANÍCIE DE INUNDAÇÃO DO ALTO RIO PARANÁ
}

\author{
INFLUENCE OF TOPOGRAPHY AND CANOPY OPENING IN THE STRUCTURE OF A HERB \\ SHRUB COMPONENT IN TWO FOREST AREAS, IN THE UPPER PARANÁ RIVER FLOODPLAIN
}

\author{
Marcos Paulo da Cruz ${ }^{1}$ João Batista Campos² José Marcelo Domingues Torezan ${ }^{3}$
}

\begin{abstract}
RESUMO
As florestas secundárias têm se expandido significativamente nos últimos anos. Dada a importância que as mesmas têm assumido nos planos de restauração e conservação, um entendimento mais abrangente sobre sua organização e funcionamento, levando em consideração as diferentes formas de vida, torna-se necessário. A fim de comparar a composição e estrutura do componente herbáceo-arbustivo entre dois fragmentos florestais com diferentes idades (RFID- remanescente florestal e FLT- floresta de 57 anos), bem como a influência relativa da topografia e abertura do dossel na organização da comunidade, dados vegetacionais e ambientais foram obtidos em 24 parcelas contíguas em cada área. Utilizaram-se correlogramas I de Moran e testes de Mantel parcial para verificar a correlação entre dados da comunidade e variáveis ambientais. Para testar diferenças entre as áreas amostrais aplicaram-se testes t de Student. As lianas mostraram-se espacialmente estruturadas (agregadas) em ambos os ambientes com um padrão semelhante ao observado para cota média. No RFID, as distâncias de cota média correlacionaram-se positivamente com as distâncias das matrizes, densidade de arbóreas e riqueza total e negativamente com as distâncias da matriz densidade de herbáceas, na FLT correlações significativas foram observadas somente entre as distâncias da matriz, densidade de arbóreas e as distâncias de desnível máximo e entre distâncias dos dados de cota e distâncias da matriz de riqueza total. Somente o componente arbustivo e abertura do dossel não apresentaram diferença significativa entre as duas áreas. A estrutura do estrato herbáceo-arbustivo tem se mostrado associada mais fortemente às características topográficas que à abertura do dossel.
\end{abstract}

Palavras-chave: floresta secundária; formas de vida; regeneração; sucessão secundária.

\begin{abstract}
Secondary forest area enlarged significantly in the recent years. Due to the importance that this kind of forest has taken regarding plans for restoration and conservation, a wider understanding of its organization and functioning, considering the different life forms is necessary. Aiming at comparing the composition and structure of the herb-shrub component between two forest areas at different stages of recovery (RFIDforest remnant and FLT-57-year old forest), as well as the relative influence of the topography and canopy opening on the organization of the community, vegetation and environmental data were collected on 24 contiguous plots in each site. Moran's I correlograms and partial Mantel tests were used to test for correlations between community data and environmental variables. To examine differences between sample areas, Student's t-tests were applied. The lianas proved to be spatially structured in both environments, with a trend similar to that observed in the average elevation. In RFID, average elevation distances were positively correlated with the distances of tree density and total richness matrices and negatively correlated

1 Biólogo, MSc., Universidade Estadual de Maringá, Av. Colombo, 5790, Zona 7, CEP 87020-900, Maringá (PR), Brasil.cruz-p2003@ig.com.br

2 Engenheiro Agrônomo, Dr., Professor/orientador credenciado no Programa de Pós-Graduação em Ecologia de Ambientes Aquáticos Continentais, Universidade Estadual de Maringá, Av. Colombo, 5790, Zona 7, CEP 87020900, Maringá (PR), Brasil. joaobatista@iap.pr.gov.br

3 Biólogo, Dr., Professor do Departamento de Biologia Animal e Vegetal, Universidade Estadual de Londrina, Rod. Celso Garcia Cid, Pr 445 Km 380, CEP 86057-970, Londrina (PR), Brasil. torezan@uel.br
\end{abstract}

Recebido para publicação em 28/02/2012 e aceito em 10/02/2017

Ci. Fl., v. 28, n. 1, jan.- mar., 2018 
with the distance matrix density of herbaceous plants. In FLT, significant correlations were observed only between the distances of the tree density matrix and those of maximum elevation difference and between distances of elevation and those of total species richness. By comparing the two areas, only the shrub components and to canopy opening did not presented differences. The structure of the herbaceous shrub component was associated with topographic characteristics, suggesting a strong influence of the flooding regime.

Keywords: secondary forest; life forms; regeneration; secondary succession.

\section{INTRODUÇÃO}

Nas últimas décadas as florestas tropicais têm experimentado uma crescente degradação devido à exploração para fim agropecuário e demais tipos de utilização da terra. Essas transformações têm atingido hot-spots de biodiversidade, com uma taxa de desflorestamento de aproximadamente três milhões de hectares por ano em toda sua extensão (ACHARD et al., 2002). Entre os anos de 2008 e 2009, a Mata Atlântica sofreu uma redução de $248 \mathrm{~km}^{2}$ em sua extensão, restando aproximadamente $22,23 \%$ de sua vegetação original (BRASIL, 2012). De maneira geral, após a degradação das terras utilizadas pelo sistema agropecuário, essas são abandonadas e, através da regeneração natural, essas áreas são ocupadas por florestas secundárias (BROWN; LUGO, 1990).

Devido ao aumento significativo das florestas secundárias, vários trabalhos foram desenvolvidos visando ao entendimento dos processos estruturais e funcionais das mesmas (NICOTRA; CHAZDON; IRIARTE, 1999; CHAZDON, 2003; PEÑA-CLAROS, 2003), haja visto que as florestas secundárias vêm assumindo grande importância ambiental devido a sua contribuição para a absorção de carbono da atmosfera, controle da erosão, recuperação e conservação da biodiversidade (NEPSTAD et al., 1996).

De um modo geral, a sucessão secundária pode ser entendida como um processo bastante complexo, no qual a ação de diversos fatores é determinante das características funcionais e estruturais da vegetação ao longo de cada estádio sucessional (BROWN; LUGO, 1990; FINEGAN et al., 1996; DENSLOW; GUZMAN, 2000). Dentre os diversos fatores que influenciam a organização da comunidade, merecem destaque as condições de luminosidade, as propriedades do solo e a disponibilidade de nutrientes.

Denslow (1980) apontou a luz como um dos principais fatores que afetam o estabelecimento e crescimento das plantas, atribuindo-lhe importância fundamental na estruturação das florestas tropicais. As condições de luminosidade apresentam um comportamento dinâmico em florestas, sendo que Denslow e Guzman (2000) registraram uma alta frequência de microssítios com níveis de luz intermediários em floresta secundária ao passo que em floresta madura foi observado um grande número de microssítios com baixo e com elevado níveis de luminosidade. Essas diferenças nas condições de luminosidade refletem na existência de distintos ambientes dentro da floresta que são explorados por grupos de espécies com diferentes capacidades de sobrevivência nesses locais (GANDOLFI, 2003).

Processos locais controlam a riqueza e a diversidade de espécies, (BOTREL et al., 2002; DALANESI; OLIVEIRA-FILHO; FONTES, 2004; GIEHL; JARENKOW, 2008). Nesse sentido, Budke, Jarenkow e Oliveira-Filho (2007) encontraram dados que apontam que a densidade de espécies se correlaciona com a topografia e com os regimes de inundação.

Aspectos topográficos em escala de paisagem afetam decisivamente a estruturação de comunidades vegetais (COSTA; MAGNUSSON; LUIZA, 2005), no entanto, variações topográficas em escala fina também são determinantes da organização da vegetação (TUOMISTO; POUSEN, 2000), haja visto que a variabilidade microtopográfica é condicionante de diferenças locais quanto à disponibilidade de água e nutrientes (AIBA; KITAYAMA; TAKYU, 2004).

Embora muitos trabalhos tenham sido desenvolvidos sobre a vegetação da planície do Alto Rio Paraná (CAMPOS; ROMAGNOLO; SOUZA, 2000; CAMPOS; SOUZA, 2002; 2003; CAMPOS; DICKINSON, 2005; CHAPLA; CAMPOS, 2011; CRUZ; CAMPOS, 2013; 2015), estudos complementares são necessários para um melhor entendimento dos fatores que influenciam a organização da comunidade vegetal nesse ambiente, sobretudo aqueles que procuram incluir todas as formas de vida.

Tendo em vista que o reconhecimento de padrões espaciais e o entendimento dos fatores que atuam sobre os processos de regeneração são essenciais para a descrição da dinâmica florestal com implicações 
para a tomada de medidas conservacionista, o presente trabalho tem por objetivo verificar a composição e a abundância da vegetação do estrato herbáceo-arbustivo em dois trechos em diferentes fases de regeneração na Planície de Inundação do Alto Rio Paraná e verificar a influência da luminosidade e da topografia na distribuição de abundância de seus componentes. Para tanto, serão utilizadas como guia as seguintes hipóteses: a) As áreas em estudo apresentam diferenças estruturais significativas, sendo que parte dessa variação é explicada pelas diferentes condições de luminosidade e parte é devida à heterogeneidade topográfica; b) As diferentes formas de vida se associam de maneira independente às variáveis ambientais, sendo esperados padrões de organização diferenciados para cada componente em cada área amostral.

\section{MATERIAL E MÉTODOS}

\section{Área de estudo}

A referida pesquisa foi desenvolvida em dois fragmentos de floresta, localizados na Planície de Inundação do Alto Rio Paraná. Um dos fragmentos é uma floresta de 57 anos (FLT), localizada no município de Porto Rico, Paraná, a $53^{\circ} 17^{\prime} \mathrm{W}, 22^{\circ} 45^{\prime} \mathrm{S}$ e altitude em torno de $232 \mathrm{~m}$. O outro fragmento é um remanescente florestal (RFID) sem idade definida, mas de acordo com fotografias aéreas históricas, em 1952 apresentava vegetação intacta e não foi impactada pesadamente nos anos subsequentes, localizada na margem direita do Rio Paraná, Mato Grosso do Sul a 53 $14^{\prime} \mathrm{W}, 22^{\circ} 43^{\prime} \mathrm{S}$ com altitude de $236 \mathrm{~m}$ (Figura 1).

A vegetação da região é classificada como Floresta Estacional Semidecidual Aluvial e está inserida no bioma Mata Atlântica. O clima, de acordo com a classificação de Köppen, é do tipo Cfa (sem estação seca definida), com temperatura média anual de $22^{\circ} \mathrm{C}$ e precipitação média anual de $1.500 \mathrm{~mm}$ (MAACK, 2002).

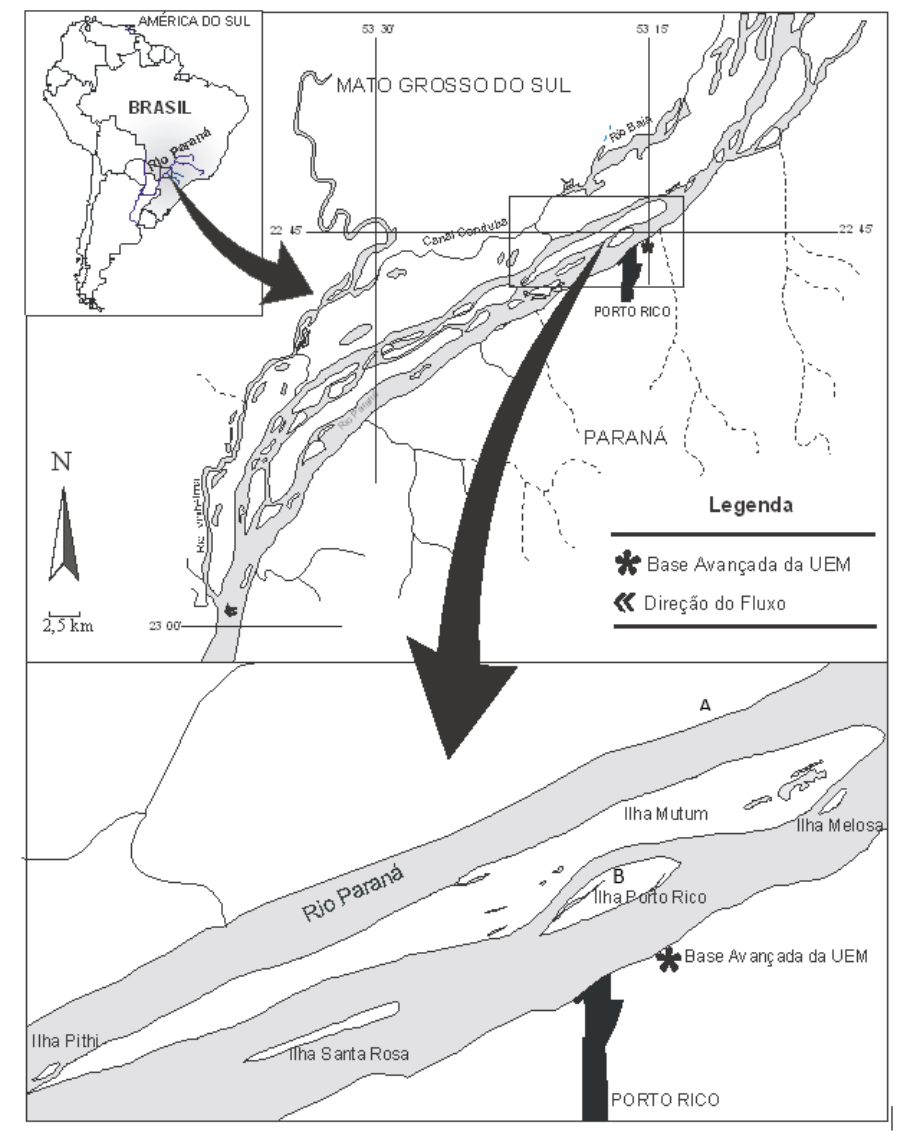

FIGURA 1: Área de estudo - Planície de inundação do Alto Rio Paraná. A: remanescente florestal (RFID); B: floresta de 57 anos (FLT).

FIGURE 1: Study area - Upper Parana River floodplain. A: Forest remnant (RFID); B: a 57-year old forest (FLT). 


\section{Delineamento amostral}

Em cada fragmento (RFID e FLT) foi delimitada uma área amostral de $60 \times 40 \mathrm{~m}$ dividida em 24 parcelas contíguas de $10 \times 10 \mathrm{~m}$. A área da FLT foi alocada com o lado maior paralelo a um Ressaco que corta a ilha, com distância de aproximadamente $15 \mathrm{~m}$ deste, e a área do RFID foi disposta com uma configuração semelhante, porém, o lado de maior tamanho foi disposto paralelo a uma lagoa natural. As parcelas de $10 \times 10 \mathrm{~m}$ foram utilizadas somente para levantamento de dados topográficos, sendo sorteado um dos vértices de cada parcela para instalação de subparcelas de $3 \times 3 \mathrm{~m}$ para levantamento de dados da vegetação e de luminosidade.

\section{Levantamento da vegetação}

Em cada subparcela foi realizado o levantamento dos indivíduos do estrato herbáceo-arbustivo. O conceito de estrato herbáceo-arbustivo que será adotado nesse trabalho corresponde àquele adotado por Richard (1996), o qual inclui plantas variando de poucos centímetros a um metro ou mais e inclui jovens árvores (serão incluída nesse trabalho espécies arbóreas com altura mínima de $10 \mathrm{~cm}$ até aquelas com circunferência máxima de $15 \mathrm{~cm}$ a $1,30 \mathrm{~m}$ do solo), arbustivas, herbáceas e lianas. A classificação dos indivíduos por forma de vida seguiu a definição de Souza, Cislinski e Romagnolo (1997). O levantamento foi feito nos meses de março e abril de 2009. O material foi identificado no campo e por meio de consulta a literatura especializada, seguindo o sistema Angiosperm Phylogeny Group III (2009). As lianas, em função das condições do material coletado, sobretudo pela ausência de estruturas reprodutivas, foram identificadas somente como morfoespécies.

\section{Dados ambientais}

O levantamento topográfico foi realizado utilizando-se nível de mangueira em régua graduada, sendo mensurada a distância vertical de cada um dos quatro vértices de cada parcela (elevação em relação ao nível do rio), posteriormente, foi estimada a cota média para cada parcela (obtidas através da média das distâncias verticais) e desnível máximo (diferença entre a maior e a menor distância vertical), o mesmo procedimento foi adotado para as duas áreas amostrais.

A abertura do dossel foi estimada a partir de fotografias hemisféricas obtidas a 1,3 $\mathrm{m}$ do solo no centro de cada subparcela. Para a tomada das fotografias foi utilizada uma câmera Nikon acoplada a uma lente "olho de peixe" com abertura angular de $180^{\circ}$, sendo a câmera montada em um tripé com nível de bolha e bússola. Foram obtidas duas séries de fotografias: Uma em março de 2009 e outra em setembro de 2009, a fim de caracterizar a abertura do dossel florestal em períodos estacionais distintos. Como não foi observada diferença significativa entre os dois períodos $(t=1,23 \alpha=0,05)$, utilizou-se a abertura do dossel dado pela média das duas séries fotográficas. As fotografias foram analisadas através do programa GLA (Gap Light Analyser) (FRAZER; CANHAM; LERTZMAN, 1999).

\section{Análise dos dados}

Para verificar se houve variação no número de indivíduos por forma de vida, bem como diferenças nas variáveis ambientais entre as duas áreas, foram aplicados testes " $\mathrm{t}$ " de Student para amostras dependentes (SOKAL; ROHLF, 1984). Esse teste foi escolhido por ser robusto face à violação do pressuposto da normalidade, mesmo com amostras pequenas. Assim, as consequências da não normalidade dos dados afeta minimamente os erros de tipo I e tipo II envolvidos na decisão. Foram produzidas curvas de rarefação para comparar a riqueza de espécies entre as diferentes formas de vida dentro e entre as áreas amostradas.

As estruturas espaciais das variáveis ambientais e dos dados da comunidade vegetal foram analisadas através de correlogramas $I$ de Moran, a fim de verificar a autocorrelação no espaço (LEGENDRE; FORTIN, 1989). Os correlogramas foram construídos com dez classes de distância (obtidas a partir da matriz de distância euclidiana das unidades amostrais) com amplitude de cinco metros. Foi criada uma matriz de distâncias euclidianas a partir das coordenadas espaciais das parcelas (As coordenadas espaciais das 24 
parcelas de cada área foram criadas no Passage v.1.1 (ROSENBERG, 2001), iniciando com a coordenada 1 nos eixos x e y e escala de 10 metros em cada parcela). Adicionalmente, foram construídas matrizes de distância euclidiana para o número de indivíduos de cada forma de vida, riqueza total de espécies (incluindo todas as formas de vida), diversidade total ( $\left.\mathrm{H}^{\prime}\right)$, cota média, desnível máximo e abertura do dossel. As correlações entre as matrizes de distância de cada área amostral foram testadas através de testes de Mantel parcial (LEGENDRE; FORTIN, 1989) usando o Passage v.1.1 (ROSENBERG, 2001). Para os cálculos das significâncias dos testes de Mantel foram utilizados testes de Monte Carlo com 9.999 permutações.

\section{RESULTADOS}

Os dados ambientais obtidos apontam que as diferentes áreas amostrais apresentam formas de elevação semelhante (gradiente altitudinal), sendo os menores valores para cota média encontrados nas parcelas próximas aos corpos de água e um aumento na cota com o distanciamento dos mesmos. Quanto à declividade, no RFID, as parcelas mais íngremes foram aquelas mais distantes da lagoa, enquanto que na FLT uma maior declividade foi observada nas parcelas do centro da área amostral.

Quanto à luminosidade constatou-se menor variação na abertura do dossel na FLT, cujo índice de abertura se mostrou pouco variável entre as unidades amostrais $(34,04 \% \pm 1,60 \%)$. Um caso um tanto diferente foi observado no RFID, que apresentou considerável variação na abertura do dossel dentro da área amostral $(35,33 \% \pm 3,05 \%)$, revelando algumas unidades amostrais com baixo e outras com elevados índices de abertura $(6,96 \%$ e $20,62 \%$, respectivamente). Foram detectadas diferenças significativas para a cota média e desnível máximo entre as áreas amostrais, sendo registrada cota altimétrica mais elevada no RFID (supracitado). No entanto, a variável abertura do dossel não se mostrou significativamente diferente entre os dois ambientes (Tabela 1).

TABELA 1: Teste $t$ de Student para o número de indivíduos da comunidade vegetal e variáveis ambientais das duas áreas amostradas na Planície de Inundação do Alto Rio Paraná.

TABLE 1: Test $t$ Student for the number of individuals of vegetal community and environments variables of two sampled areas in upper Paraná River floodplain.

\begin{tabular}{|c|c|c|c|c|c|c|c|}
\hline \multirow{2}{*}{ Componentes } & \multicolumn{2}{|c|}{ Média } & \multicolumn{2}{|c|}{$\mathrm{S}$} & \multirow{2}{*}{$\mathrm{t}$} & \multicolumn{2}{|c|}{$p$} \\
\hline & FLT & RFID & FLT & RFID & & & \\
\hline Arbóreas & 15,875 & 31,958 & 11,380 & 12,319 & 6,029 & 0,000 & $*$ \\
\hline Arbustivas & 8,500 & 10,292 & 4,899 & 9,448 & 0,904 & 0,375 & ns \\
\hline Herbáceas & 9,917 & 23,083 & 13,164 & 25,358 & 2,942 & 0,007 & $*$ \\
\hline Lianas & 5,208 & 24,375 & 5,778 & 16,428 & 5,052 & 0,000 & * \\
\hline Abertura dossel (\%) & 34 & 35,3 & 0,028 & 0,025 & 0,731 & 0,472 & ns \\
\hline Cota média $(\mathrm{cm})$ & 210,00 & 359,17 & 23,960 & 18,768 & 22,19 & 0,000 & * \\
\hline Desnível máx. (cm) & 65,458 & 30,042 & 22,507 & 14,352 & $-5,949$ & 0,000 & $*$ \\
\hline
\end{tabular}

Em que: $\mathrm{FLT}=$ Floresta de 57 anos; RFID $=$ Remanescente florestal; Média $=$ número de indivíduos dividido pelo total de subparcelas; $\mathrm{S}=$ Desvio padrão; $*$ = Significativo; $\mathrm{ns}=$ Não significativo $(\alpha=0,05) ; p=$ significância.

No RFID observou-se uma predominância das arbóreas, com 767 indivíduos (35,62\%), seguido pelas lianas com 585 indivíduos (27,17\%), herbáceas com 554 indivíduos (25,73\%) e arbustivas com 247 indivíduos (11,47\%). Na FLT foram amostrados 381 indivíduos $(40,19 \%)$ pertencentes às arbóreas, seguido pelas herbáceas, 238 indivíduos (25,11\%), arbustivas, 204 indivíduos $(21,52 \%)$ e lianas, 125 indivíduos $(13,18 \%)$. Exceto para o grupo das arbustivas, todas as demais formas de vida diferem significativamente quanto ao número de indivíduos entre as áreas amostradas (Tabela 1).

Considerando o conjunto das duas áreas, foram amostrados 3.101 indivíduos, 61 espécies, 47 gêneros e 26 famílias. Do total de indivíduos amostrados, foram observados 2.153 indivíduos no RFID e 948 indivíduos na FLT. Das 61 espécies amostradas, 25 espécies (40,98\%) foram comuns aos dois ambientes, 11 espécies (18,03\%) foram exclusivas da FLT e 25 espécies (40,98\%) foram encontradas somente no RFID (Tabela 2). 
TABELA 2: Lista das espécies encontradas nas duas áreas amostrais e seus respectivos números de indivíduos. RFID = Remanescente florestal.

TABLE 2: $\quad$ List of species founds in two sampled area and respective number of individual.

\begin{tabular}{|c|c|c|c|}
\hline \multirow[b]{2}{*}{ Espécies } & \multirow[b]{2}{*}{ FV } & \multicolumn{2}{|c|}{ Número de indivíduos } \\
\hline & & RFID & FLT de 57 anos \\
\hline Albizia hasslerii (chodat) Burkart & $\mathrm{Ab}$ & 23 & 57 \\
\hline Allophylus edulis Radlk. Ex warm & $\mathrm{Ab}$ & 37 & 1 \\
\hline Cecropia pachystachya Tréc. & $\mathrm{Ab}$ & 1 & 0 \\
\hline Celtis iguanaea (Jacq.) Sarg. & $\mathrm{Ab}$ & 0 & 9 \\
\hline Citrus limon (L.) Burm. F. & $\mathrm{Ab}$ & 0 & 2 \\
\hline Coussarea platyphylla M. Arg. & $\mathrm{Ab}$ & 105 & 4 \\
\hline Eugenia florida DC. & $\mathrm{Ab}$ & 22 & 10 \\
\hline Eugenia egensis DC. & $\mathrm{Ab}$ & 79 & 6 \\
\hline Eugenia hiemalis Cambess & $\mathrm{Ab}$ & 83 & 7 \\
\hline Garcinia gardneriana (P1.et Tr.) & $\mathrm{Ab}$ & 90 & 0 \\
\hline Genipa americana $\mathrm{L}$. & $\mathrm{Ab}$ & 6 & 14 \\
\hline Guarea macrophylla Vahl & $\mathrm{Ab}$ & 0 & 1 \\
\hline Inga vera Willd. & $\mathrm{Ab}$ & 60 & 3 \\
\hline Nectandra falcifolia (Nees.) Cast. Ex Mez. & $\mathrm{Ab}$ & 0 & 3 \\
\hline Nectandra mollis (Kunth) Nees & $\mathrm{Ab}$ & 47 & 0 \\
\hline Ocotea diospyrifolia (Meissn.) Mez. & $\mathrm{Ab}$ & 12 & 1 \\
\hline Peltophorum dubium (Spreng) Taub & $\mathrm{Ab}$ & 4 & 0 \\
\hline Picramnia sellowii Planch & $\mathrm{Ab}$ & 0 & 79 \\
\hline Protium heptaphyllum & $\mathrm{Ab}$ & 57 & 0 \\
\hline Annona emarginata (Schltdl) H. Rainer & $\mathrm{Ab}$ & 0 & 3 \\
\hline Sapindus saponaria L. & $\mathrm{Ab}$ & 0 & 1 \\
\hline Senna alata (L.) Roxb. & $\mathrm{Ab}$ & 9 & 0 \\
\hline Sloanea garckeana k. Schum. & $\mathrm{Ab}$ & 0 & 4 \\
\hline Sloanea guianenses (Aubl.) Bentham. & $\mathrm{Ab}$ & 21 & 0 \\
\hline Spondias lutea L. & $\mathrm{Ab}$ & 1 & 0 \\
\hline Tabernaemontana catharinensis A.DC. & $\mathrm{Ab}$ & 17 & 59 \\
\hline Trichilia pallida $\mathrm{Sw}$. & $\mathrm{Ab}$ & 56 & 12 \\
\hline Triplaris americana $\mathrm{L}$. & $\mathrm{Ab}$ & 6 & 103 \\
\hline Unonopsis lindmanii R.E.Fr. & $\mathrm{Ab}$ & 109 & 1 \\
\hline Zygia cauliflora (Willd.) Killip. & $\mathrm{Ab}$ & 8 & 0 \\
\hline Morfoespécie 1 & Abt & 61 & 0 \\
\hline Piper tuberculatum Jacq & Abt & 21 & 9 \\
\hline Pisonia aculeata $\mathrm{L}$. & Abt & 7 & 0 \\
\hline Psychotria capilaris Jacq & Abt & 141 & 26 \\
\hline Psychotria carthagenensis jacq. & Abt & 17 & 169 \\
\hline Adiantum latifolium Lam. & $\mathrm{He}$ & 316 & 0 \\
\hline Commelina sp & $\mathrm{He}$ & 2 & 0 \\
\hline Commelina diffusa burm.F. & $\mathrm{He}$ & 5 & 184 \\
\hline Oeceoclades maculata (Lindl.) Lindl. & $\mathrm{He}$ & 24 & 1 \\
\hline Panicum sp & $\mathrm{He}$ & 206 & 3 \\
\hline Solanum $s p$ & $\mathrm{He}$ & 1 & 50 \\
\hline Morfoespécie 2 & $\mathrm{Li}$ & 499 & 0 \\
\hline
\end{tabular}


TABELA 2: Continuação...

TABLE 2: Continued...

\begin{tabular}{|c|c|c|c|}
\hline \multirow[b]{2}{*}{ Espécies } & \multirow[b]{2}{*}{ FV } & \multicolumn{2}{|c|}{ Número de indivíduos } \\
\hline & & RFID & FLT de 57 anos \\
\hline Morfoespécie 3 & $\mathrm{Li}$ & 31 & 0 \\
\hline Morfoespécie 4 & $\mathrm{Li}$ & 8 & 0 \\
\hline Morfoespécie 5 & $\mathrm{Li}$ & 1 & 0 \\
\hline Morfoespécie 6 & $\mathrm{Li}$ & 1 & 0 \\
\hline Morfoespécie 7 & $\mathrm{Li}$ & 1 & 18 \\
\hline Morfoespécie 8 & $\mathrm{Li}$ & 5 & 0 \\
\hline Morfoespécie 9 & $\mathrm{Li}$ & 2 & 0 \\
\hline Morfoespécie 10 & $\mathrm{Li}$ & 8 & 0 \\
\hline Morfoespécie 11 & $\mathrm{Li}$ & 3 & 0 \\
\hline Morfoespécie 12 & $\mathrm{Li}$ & 2 & 0 \\
\hline Morfoespécie 13 & $\mathrm{Li}$ & 3 & 0 \\
\hline Morfoespécie 14 & $\mathrm{Li}$ & 6 & 0 \\
\hline Morfoespécie 15 & $\mathrm{Li}$ & 0 & 9 \\
\hline Morfoespécie 16 & $\mathrm{Li}$ & 0 & 1 \\
\hline Paullinia elegans Cambess. & $\mathrm{Li}$ & 1 & 42 \\
\hline Scleria pterota Presl. & $\mathrm{Li}$ & 12 & 33 \\
\hline Serjania $s p$ & $\mathrm{Li}$ & 2 & 3 \\
\hline Smilax campestris Gris. & $\mathrm{Li}$ & 0 & 19 \\
\hline Total & & 2153 & 948 \\
\hline
\end{tabular}

Em que: $\mathrm{FLT}=$ Floresta de 57 anos; $\mathrm{FV}=$ Forma de vida; $\mathrm{Ab}=$ Arbóreas; $\mathrm{Abt}=$ Arbustivas; $\mathrm{He}=$ Herbáceas; $\mathrm{Li}=$ Lianas.

No RFID, as lianas apresentaram autocorrelação espacial positiva a curta distância e negativa em distâncias maiores, representação típica de gradiente (padrão agregado). Dentro da mesma área, todas as demais formas de vida não apresentaram dependência espacial na escala de estudo considerada (Figura 2A). As variáveis ambientais cota média e desnível máximo apresentaram autocorrelação espacial com alcance na dependência espacial semelhantes entre si e com aquele apresentado pelas lianas (Figura 2C). Na FLT, de maneira semelhante ao RFID, dos componentes da vegetação, somente as lianas estiveram espacialmente estruturadas (padrão agregado) (Figura 2B), com um alcance na dependência espacial semelhante ao apresentado pela cota média (Figura 2C). As variáveis ambientais desnível máximo e abertura do dossel não se mostraram espacialmente autocorrelacionadas na FLT (padrão aleatório).

Considerando os dois ambientes, foram amostradas 31 espécies pertencentes ao grupo das arbóreas, cinco arbustivas, 19 lianas e seis herbáceas. As curvas de riqueza geradas por rarefação demonstraram que, entre os ambientes amostrados, a riqueza diferiu somente para o grupo das lianas e das arbustivas,

Considerando os dois ambientes, foram amostradas 31 espécies pertencentes ao grupo das arbóreas, cinco arbustivas, 19 lianas e seis herbáceas. As curvas de riqueza geradas por rarefação demonstraram que, entre os ambientes amostrados, a riqueza diferiu somente para o grupo das lianas e das arbustivas, sendo observados níveis mais elevados para os dois grupos no RFID. 

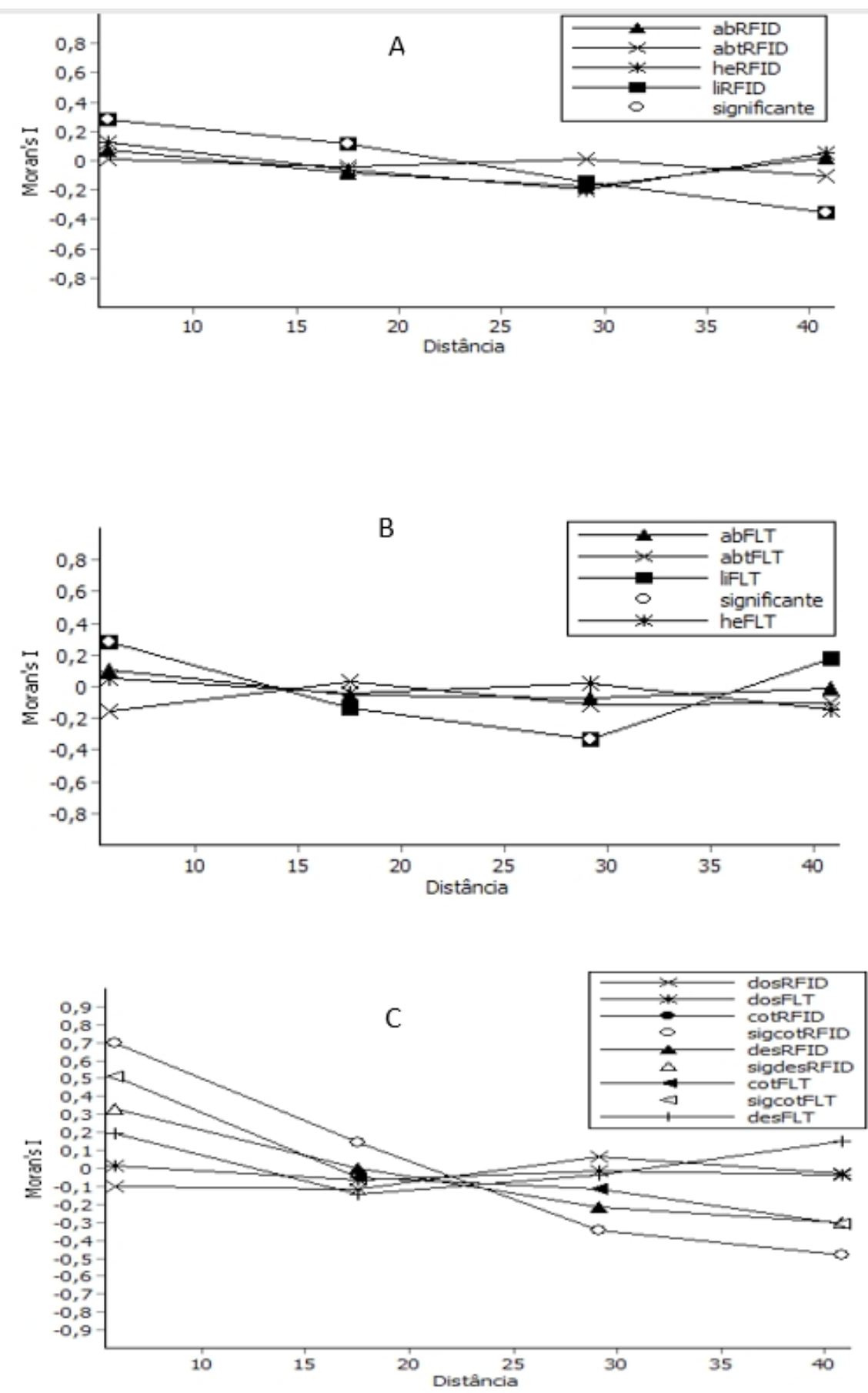

FIGURA 2: Correlogramas I de Moran para os componentes, densidade da vegetação (A e B) e variáveis ambientais (C), em cada área amostral. Ab - arbóreas; abt - arbustivas; He - herbáceas; li - lianas; dos - abertura do dossel; cot - cota média; des - desnível máximo; RFID - remanescente florestal; FLT - floresta de 57 anos; sigcotRFID - cota média remanescente florestal significativo; sigdesRFID - desnível máximo remanescente florestal significativo; sigcotFLT - cota média floresta de 57 anos significativo.

FIGURE 2: Moran I Correlograms for density of vegetation (A e B) and environment variables (C), in each sample area. Ab - trees; abt - shrub; He - herbs; li - lianas; dos - openness canopy; cot - average level; des - highest unevenness; RFID - forest remnant; FLT - 57-year-old forest; sigcotRFID - significative average level forest remnant; sigdesRFID - significative highest unevenness forest remnant; sigcot FLT - significative. 
Ao se considerar cada ambiente isoladamente, foi observado um padrão semelhante entre os mesmos, em que se evidencia maior riqueza para as arbóreas, seguido pelas lianas e por último herbáceas e arbustivas que apresentam níveis de riqueza semelhantes (Figura 3).

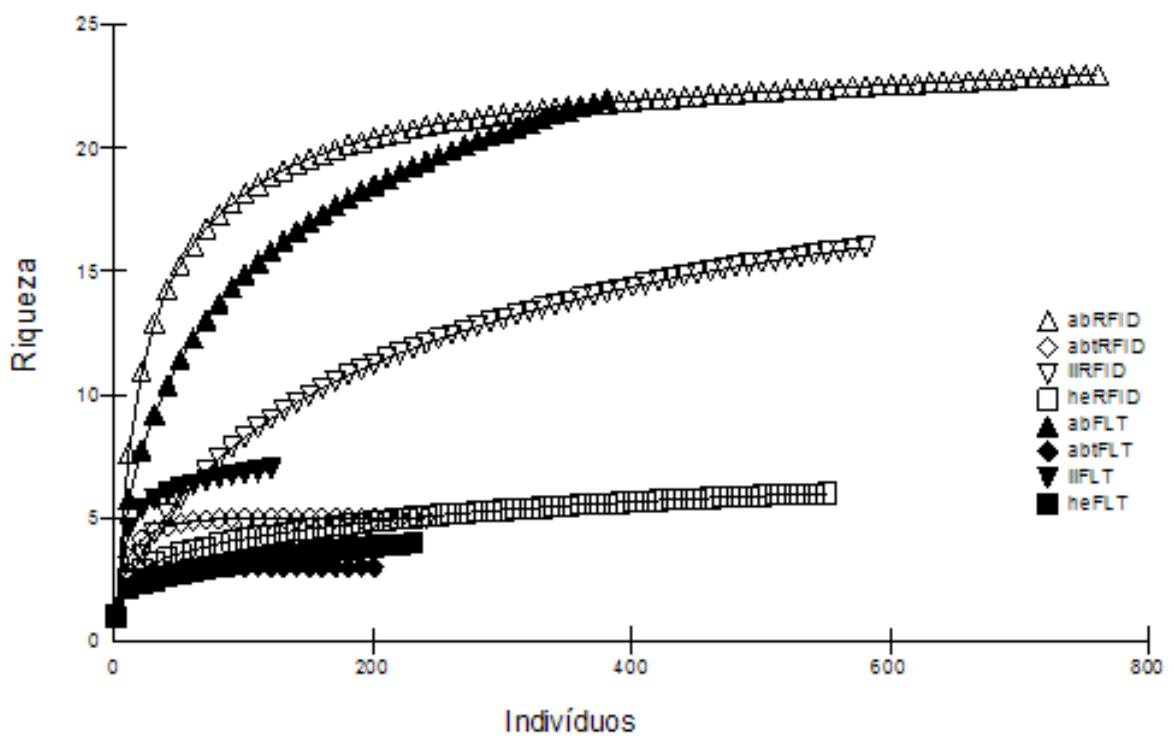

FIGURA 3: Curvas de rarefação geradas para as diferentes formas de vida em cada uma das áreas amostrais. Ab arbóreas; abt - arbustivas; He - herbáceas; li - lianas; RFID - remanescente florestal; FLT - floresta de 57 anos.

FIGURE 3: Rarefaction curves to generate for different life forms in each sampled area. Ab - trees; abt - shrub; He - herbs; li - lianas; RFID - forest remnant; FLT - 57-year old forest.

Os testes parciais de Mantel (Tabela 3) demonstraram que no RFID as distâncias de cota média apresentaram-se positivamente correlacionadas com as matrizes de distâncias de abundância de arbóreas e riqueza total. Os demais componentes da vegetação não se mostraram significativamente correlacionados com nenhuma das variáveis ambientais.

Situação um pouco diferente foi observada na FLT, na qual correlações significativamente positivas foram evidenciadas somente entre a matriz de distâncias de abundância das arbóreas e as distâncias de desnível máximo e entre as distâncias de riqueza total e cota média.

TABELA 3: Resultados dos testes de Mantel parcial para correlação entre as variáveis cota média, desnível máximo, abertura do dossel, abundância de arbóreas, arbustivas, herbáceas e lianas, riqueza total e diversidade $\left(\mathrm{H}^{\prime}\right)$, para os dois ambientes amostrados (FLT - floresta de 57 anos, RFID - remanescente florestal).

TABLE 3: Partial Mantel test result for the correlation between variables average level, highest unevenness, openness of the canopy, abundance of trees, shrub, herb and lianas, total richness and diversity $\left(\mathrm{H}^{\prime}\right)$, for the two environments sampled (FLT- 57-year-old forest, RFID- forest remnant).

\begin{tabular}{ccccc}
\hline Matriz 1 & Matriz 2 & Matriz 3 (constante) & Correlação & $\mathrm{p}<0,01$ \\
\hline Arbóreas RFID & Cota média & Distância & 0,15835 & 0,00431 \\
Arbóreas RFID & Desn. Máx. & Distância & 0,03471 & 0,64260 \\
Arbóreas RFID & Abert. Doss. & Distância & $-0,06929$ & 0,53150 \\
Arbustivas RFID & Cota média & Distância & 0,01585 & 0,85840 \\
Arbustivas RFID & Desn. Máx. & Distância & $-0,13238$ & 0,14280 \\
Arbustivas RFID & Abert. Doss. & Distância & $-0,06772$ & 0,63090 \\
Lianas RFID & Cota média & Distância & $-0,00014$ & 0,99800 \\
Lianas RFID & Desn. Máx. & Distância & 0,03987 & 0,58410 \\
Herbáceas RFID & Cota média & Distância & $-0,17552$ & 0,00642 \\
\hline
\end{tabular}


TABELA 3: Continuação...

TABLE 3: Continued...

\begin{tabular}{|c|c|c|c|c|}
\hline Matriz 1 & Matriz 2 & Matriz 3 (constante) & Correlação & $\mathrm{p}<0,01$ \\
\hline Herbáceas RFID & Desn. Máx. & Distância & $-0,11108$ & 0,22500 \\
\hline Herbáceas RFID & Abert. Doss. & Distância & $-0,12624$ & 0,25190 \\
\hline Arbóreas FLT & Cota média & Distância & $-0,04765$ & 0,66280 \\
\hline Arbóreas FLT & Desn. Máx. & Distância & 0,18341 & 0,00137 \\
\hline Arbóreas FLT & Abert. Doss. & Distância & $-0,12579$ & 0,20160 \\
\hline Arbustivas FLT & Cota média & Distância & $-0,08815$ & 0,47690 \\
\hline Arbustivas FLT & Desn. Máx. & Distância & $-0,08938$ & 0,56710 \\
\hline Arbustivas FLT & Abert. Doss. & Distância & $-0,12305$ & 0,33797 \\
\hline Lianas FLT & Cota média & Distância & 0,02047 & 0,84390 \\
\hline Lianas FLT & Desn. Máx. & Distância & $-0,14549$ & 0,24260 \\
\hline Lianas FLT & Abert. Doss. & Distância & $-0,01869$ & 0,88220 \\
\hline Herbáceas FLT & Cota média & Distância & $-0,02362$ & 0,85310 \\
\hline Herbáceas FLT & Desn. Máx. & Distância & 0,06338 & 0,67730 \\
\hline Herbáceas FLT & Abert. Doss. & Distância & $-0,06434$ & 0,57730 \\
\hline Diversidade RFID & Cota média & Distância & 0,01662 & 0,83970 \\
\hline Diversidade RFID & Desn. Máx. & Distância & $-0,16180$ & 0,03550 \\
\hline Diversidade RFID & Abert. Doss. & Distância & $-0,05451$ & 0,67820 \\
\hline Riqueza RFID & Cota média & Distância & 0,13244 & 0,00110 \\
\hline Riqueza RFID & Desn. Máx. & Distância & $-0,07093$ & 0,39280 \\
\hline Riqueza RFID & Abert. Doss. & Distância & 0,04132 & 0,74580 \\
\hline Diversidade FLT & Cota média & Distância & $-0,00237$ & 0,98160 \\
\hline Diversidade FLT & Desn. Máx. & Distância & $-0,13243$ & 0,28060 \\
\hline Diversidade FLT & Abert. Doss. & Distância & 0,02393 & 0,83860 \\
\hline Riqueza FLT & Cota média & Distância & 0,14337 & 0,00117 \\
\hline Riqueza FLT & Desn. Máx. & Distância & $-0,10988$ & 0,36380 \\
\hline Riqueza FLT & Abert. Doss. & Distância & $-0,06680$ & 0,50470 \\
\hline
\end{tabular}

\section{DISCUSSÃO}

Embora o tamanho da área amostral seja de pequena dimensão, $432 \mathrm{~m}^{2}$ incluindo os dois ambientes, foi amostrado um número de indivíduos relativamente grande, 3.101 indivíduos no total. Um número expressivo de espécies também foi contabilizado, totalizando 61 espécies nos dois ambientes. Em um trabalho desenvolvido na mesma região, Zviejkovski (2008) registrou 44 espécies em três áreas de diferentes estágios sucessionais, totalizando uma área amostral de três hectares. No entanto, a autora considerou apenas as arbóreas em seu estudo, incluindo somente aquelas com um metro ou mais de altura. O maior número de espécies encontrado nesse estudo, cuja área amostral é significativamente inferior, está relacionado ao fato de se considerar todas as formas de vida e também por estar incluindo indivíduos arbóreos de pequeno tamanho (tamanho mínimo $\geq$ a $10 \mathrm{~cm}$ ). Reconhecidamente, espécies arbóreas dentro dessa categoria de tamanho geralmente são encontradas em alta densidade próximas a seus parentais.

Em um plano comparativo entre os dois ambientes, diferenças nítidas são evidenciadas. Do total de indivíduos amostrados, um número superior foi encontrado no RFID, bem como, um número maior de espécies, em relação à FLT. Esse padrão, no qual ambientes com diferentes idades de recuperação apresentam diferenças marcante em sua estrutura e composição, é bastante característico e tem sido observado em diversos estudos (PICKETT; COLLINS; ARMESTO, 1987; AIDE et al., 1995; GUARIGUATA et al., 1997; NICOTRA; CHAZDON; IRIARTE, 1999; DENSLOW; GUZMAN, 2000; PEÑA-CLAROS, 2003; CHAZDON, 2003). Na maioria desses trabalhos, tais diferenças têm sido atribuídas principalmente à 
variação nas condições de luminosidade entre os ambientes. No entanto, este não foi verificado no presente estudo, dado que a abertura do dossel não apresentou diferença significativa entre as áreas amostrais.

Ao que tudo indica, a topografia parece ser o principal fator responsável pela variação na estrutura e composição da vegetação entre os ambientes analisados. Os dados obtidos têm demonstrado diferença significativa para a variável cota média entre a RFID e FLT, como essa região experimenta inundações periódicas em que o nível hidrométrico do rio varia em até três metros (THOMAZ; ROBERTO; BINI, 1997), os diferentes ambientes estão sujeitos a condições de umidade diferenciada. Nesse sentido, áreas em cotas altimétricas mais baixas estão sujeitas à maior exposição ao estresse aquático, o que pode resultar em menor riqueza e diversidade de organismos em relação às áreas com cota mais elevada (CONNELL, 1978).

A inclusão de todas as formas de vida da vegetação em estudos ecológicos tem sua importância destacada (GILLIAM; TURRIL; ADAMS, 1995; MEIRA-NETO; MARTINS, 2000; MARTINI, 2002), já que tal procedimento pode revelar padrões mais consistentes da realidade dos sistemas analisados. No presente estudo, uma análise detalhada por forma de vida tem revelado a predominância das arbóreas, tanto no número de espécies quanto nos níveis de abundância. O padrão observado está em conformidade com aquele obtido por Martini (2002) e reflete uma tendência natural, já que em áreas florestadas é esperado um número maior de arbóreas em relação às demais formas de vida.

Em relação às lianas, no RFID elas apresentaram maior abundância, bem como maior riqueza de espécies em relação à FLT. Segundo Schnitzer, Dalling e Carson (2000), as lianas apresentam preferência por ambientes bem iluminados. Embora não tenha sido detectada diferença significativa na abertura do dossel entre os dois fragmentos, os dados revelaram a existência de parcelas com elevados índices de abertura do dossel no RFID, o que pode ser indicativo de antigas clareiras, nas quais potencialmente as lianas teriam se estabelecido com sucesso, justificando assim, sua maior abundância nessa área.

Embora o número de indivíduos do componente herbáceo seja significativamente superior no RFID em relação à FLT, neste último, as herbáceas representam a segunda forma de vida mais expressiva. Como constatado por Guariguata e Ostertag (2001), existe uma tendência na redução da abundância das herbáceas ao longo da recuperação da vegetação. No entanto, essa tendência não se confirma no presente estudo, pois a floresta mais jovem (FLT) apresenta menor abundância de herbáceas em relação à floresta mais madura (RFID). Segundo Oliveira-Filho et al. (1994), variações topográficas e regimes de inundação afetam diretamente a estrutura de florestas ripárias. Dessa forma, como a FLT está em uma cota altimétrica inferior ao RFID, essa área está submetida à maior pressão dos eventos de inundação, o que possivelmente esteja limitando o desenvolvimento da comunidade como um todo.

O componente arbustivo foi o único que não apresentou diferença significativa em sua abundância entre os dois ambientes. No entanto, essa forma de vida é representada por apenas cinco espécies no total, distribuídas de modo uniforme por todas as unidades amostrais. Segundo Lytle e Poff (2004), os organismos tendem a apresentar características adaptativas diferenciadas aos eventos de cheias, o que pode justificar o padrão observado para esse grupo, dado que apenas algumas espécies teriam superado o filtro ambiental e se estabelecido com sucesso.

A sequência na riqueza de espécies por forma de vida observada segue uma tendência única para os dois ambientes, na qual é observado maior número de espécies arbóreas, seguida pelas lianas e por fim níveis semelhantes de riqueza entre herbáceas e arbustivas. Seria esperado maior riqueza do componente herbáceo em relação às demais formas de vida na FLT, por essa ser uma floresta mais jovem, e então essa ordem se inverteria no RFID que possui idade de recuperação mais avançada (GUARIGUATA; OSTERTAG, 2001). No entanto, o padrão de riqueza se mantém semelhante entre os dois ambientes. Esse fato é mais um indicativo de que a comunidade do estrato herbáceo-arbustivo está sendo fortemente influenciada pelo regime de inundação, que está atuando como uma força estruturadora agindo em escala local. Esse fenômeno possivelmente está superando os efeitos esperados pela sucessão secundária, pois, de acordo com a hipótese do distúrbio intermediário (CONNELL, 1978), comunidades que sofrem distúrbios de grande magnitude tendem a retroceder a estágios anteriores da sucessão.

A escala espacial adotada no presente estudo foi capaz de captar uma heterogeneidade ambiental em escala fina, no entanto, essa heterogeneidade tem se revelado somente para os dados da topografia (gradiente na cota média e desnível máximo). Quanto à luminosidade, alguns autores (NICOTRA; CHAZDON; IRIARTE, 1999; MONTGOMERY; CHAZDON, 2002; ENOKI; ABE, 2004; SOUZA; 
MARTINS, 2005) encontraram significativa variabilidade em escala local. Tal variabilidade pode estar associada à suscetibilidade a inundação (BIANCHINI; PIMENTA; SANTOS, 2001), porém, no presente estudo, não foi encontrada autocorrelação espacial para abertura do dossel em nenhum dos ambientes, demonstrando que as unidades amostrais apresentam dossel com um padrão espacial não estruturado.

Dos componentes da vegetação, somente as lianas se mostraram espacialmente estruturadas, apresentando uma estrutura espacial típica de gradiente. Tanto no RFID quanto na FLT, a amplitude da dependência espacial das lianas variou em níveis semelhantes aos apresentados pela cota média, no entanto, os testes de mantel não confirmaram nenhuma correlação entre lianas e cota média, de forma que não se pode inferir relação significativa entre topografia e a distribuição das lianas nos ambientes analisados.

No RFID, a matriz de distâncias das arbóreas apresentou correlação positiva com as distâncias de cota altimétrica, indicando uma estruturação desse componente da vegetação em função da variação na elevação das parcelas. Diversos estudos (OLIVEIRA-FILHO et al., 1994; FERREIRA; STOHLGREN, 1999; DAMASCENO-JUNIOR et al., 2005; BUDKE; JARENKOW; OLIVEIRA-FILHO, 2007) têm relatado a preferência das arbóreas por posições topográficas mais elevadas em florestas ribeirinhas. De modo semelhante às lianas, as plântulas arbóreas também são bastante sensíveis ao estresse causado por inundações periódicas, como no presente estudo jovens arbóreos foram incluídos, essa preferência por habitat se mostra mais pronunciada mesmo na escala de estudo considerada.

Na FLT, foi observada correlação positiva entre a matriz de distâncias de arbóreas e as distâncias de declividade, ao que tudo indica, em áreas de alta umidade, feições topográficas mais íngremes em pequena escala podem ser favoráveis ao estabelecimento de espécies do componente arbóreo, dado que tais condições do relevo favorecem o escoamento de água, reduzindo, assim, a severidade do ambiente. Esses dados corroboram os resultados obtidos por Cruz e Campos (2013), que apontam que o sucesso no estabelecimento dos indivíduos dessa forma de vida em áreas alagáveis está condicionado às condições de menor estresse hídrico.

As distâncias dos dados de riqueza total de espécies têm se mostrado positivamente correlacionadas com as distâncias de cota média. Esse resultado vai ao encontro dos dados obtidos por Chase (2007), que encontrou uma redução na riqueza de espécies em ambientes mais severos. Como as áreas de estudos estão situadas em uma região sujeita à inundação, as unidades localizadas em cotas altimétricas inferiores podem representar sítios ambientais mais extremos. Dessa maneira, os resultados observados dão suporte a um entendimento de que existe uma determinação das variáveis topográficas na estruturação da comunidade do estrato herbáceo-arbustivo.

\section{CONCLUSÃO}

Diante dos resultados obtidos pode-se chegar às seguintes conclusões:

a) Em áreas sujeitas a inundações, locais em diferentes fases de recuperação da vegetação parecem apresentar mudanças estruturais fortemente relacionadas às condições ambientais locais;

b) A topografia exerce maior influência que as condições de luminosidade na estruturação da comunidade do estrato herbáceo-arbustivo em áreas inundáveis;

c) Variações topográficas em escala fina têm se mostrado determinante das variações de riqueza total do estrato herbáceo-arbustivo e da abundância apresentada pelas arbóreas;

d) As diferentes formas de vida respondem de maneira diferenciada às variações apresentadas pelas variáveis, cota média e abertura do dossel, de modo que a importância de se considerar todas as formas de vida em estudos que buscam definir padrões gerais na organização das comunidades vegetais é reforçada.

\section{REFERÊNCIAS}

ANGIOSPERM PHYLOGENY GROUP III. An update of the Angiosperm Phylogeny Group classification for the orders and families of flowering plants: APG III. Botanical Journal of the Linnean Society, London, v. 161, n. 2, p. 105-121, 2009.

ACHARD, F. et al. Determination of deforestation rates of the worlds humid tropical forests. Science, Washington, v. 297, n. 5583, p. 999-1002, 2002. 
AIBA, S.; KITAYAMA, K.; TAKYU, M. Habitat association with topography and canopy structure of tree species in a tropical montane forest on Mount Kinabalu, Borneo. Plant Ecology, New York, v. 174, n. 1, p. 147-161, 2004.

AIDE, T. M. et al. Forest recovery in abandoned tropical pastures in Puerto Rico. Forest Ecology and Management, Amsterdam, v. 77, p. 77-86, 1995.

BIANCHINI, E.; PIMENTA, J. A.; SANTOS, F. A. M. Spatial and temporal variation in the canopy cover in a tropical semi-deciduous forest. Brazilian archives of biology and technology, Curitiba, v. 44, n. 3, p. 269-276, 2001.

BOTREL, R. T. et al. Influência do solo e topografia sobre as variações da composição florística e estrutura da comunidade arbóreo-arbustiva de uma floresta estacional semidecidual em Ingaí, MG. Revista Brasileira de Botânica, São Paulo, v. 25, n. 2, p. 195-213, 2002.

BRASIL. Ministério do Meio Ambiente. Monitoramento do desmatamento nos biomas brasileiros por satélite, acordo de cooperação técnica MMA/IBAMA: monitoramento do bioma Mata Atlântica 2008 a 2009. Brasília: MMA, 2012. 101 p. Disponível em: <http://www.mma.gov.br/estruturas/sbf_chm_rbbio/_ arquivos/relatorio_tcnico_mata_atlantica_2008_2009_72.>. Acesso em: 15 fev. 2014.

BROWN, S.; LUĞO, A. E. Tropical secondary forest. Journal of tropical ecology, Cambridge, v. 6, n. 1, p. $1-32,1990$.

BUDKE, J. C.; JARENKOW, J. A.; OLIVEIRA-FILHO, A. T. Relationships between tree component structure, topography and soils of a riverside forest, Rio Botucaraí, Southern Brazil. Plant Ecology, New York, v. 189, p. 187-200, 2007.

CAMPOS, J. B.; DICKINSON, G. Regeneração de florestas na Área de Proteção Ambiental -APA das Ilhas e Várzeas do Rio Paraná. Cadernos da Biodiversidade, Curitiba, v. 5, n.1, p. 50-59, 2005.

CAMPOS, J. B.; ROMAGNOLO, M. B.; SOUZA, M. C. Structure, composition and spatial distribution of tree species in a remnant of the semideciduous seasonal Alluvial Forest of the upper Paraná River Floodplain. Brazilian Archives of Biology and Technology, Curitiba, v. 43, n. 2, p. 185-194, 2000.

CAMPOS, J. B.; SOUZA, M. C. Arboreus vegetation of an alluvial riparian forest and their soil relations: Porto Rico Island, Paraná River, Brazil. Brazilian Archives of Biology and Technology, Curitiba, v. 45, p. 137-149, 2002.

CAMPOS, J. B.; SOUZA, M. C. Potencial for natural forest regeneration from seed bank in an Upper Paraná River Floodplain, Brazil. Brazilian Archives of Biology and Technology, Curitiba, v. 46 n. 4, p. 625-639, 2003.

CHAPLA, T. E.; CAMPOS, J. B. Soil seed bank during succession at an abandoned pasture in the upper Paraná river-floodplain, Brazil. Acta Scientiarum Biological Sciences, Maringá, v. 33, n. 1, p. 59-69, 2011. CHASE, J. M. Drought midiates the importance of stochastic community assembly. Proceedings of the National Academy of Science of the USA, Washington, v. 104, p. 17430-17434, 2007.

CHAZDON, R. L. Tropical forest recovery: legacies of human impact and natural disturbances. Perspectives in Plant Ecology, Evolution and Systematics, Amsterdam, v. 6, p. 51-71, 2003.

CONNELL, J. H. Diversity in tropical rain forest and coral reefs. Science, Washington, v. 199, n. 4335, p. 1302-1310, 1978.

COSTA, F. R. C.; MAGNUSSON, W. E.; LUIZA, R. C. Mesoscale distribution patterns of amazonian understorey herbs in relation to topography, soil and watersheds. Journal of Ecology, Hoboken, v. 92, p. 863-878, 2005.

CRUZ, M. P.; CAMPOS, J. B. Regime de inundação e a regeneração arbórea em duas áreas florestais na Planície alagável do alto Rio Paraná. Biotemas, Florianópolis, v. 26, n. 3, p. 71-82, 2013.

CRUZ, M. P.; CAMPOS, J. B. Estrutura e composição do estrato herbáceo-arbustivo em duas áreas florestais com diferentes idades de recuperação, na Planície de Inundação do Alto Rio Paraná. Ambiência, Guarapuava, v. 11, n. 2, p. 375-392, 2015.

DALANESI, P. E.; OLIVEIRA-FILHO, A. T.; FONTES, M. A. Flora e estrutura do componente arbóreo da floresta do parque ecológico quedas do rio Bonito, Lavras, MG, e correlações entre a distribuição das espécies e variáveis ambientais. Acta Botânica Brasileira, Belo horizonte, v. 18, n. 4, p. 737-757, 2004. DAMASCENO-JUNIOR, G. A. et al. Structure, distribution of species and inundation in a riparian forest of Rio Paraguai, Pantanal, Brazil. Flora, Amsterdan, v. 200, p. 119-135, 2005. 
DENSLOW, J. S. Gap partitioning among tropical rainforest tree. Biotropica: Tropical succession, Lawrence, v. 47, p. 47-55, 1980.

DENSLOW, J. S.; GUZMAN, S. Variation in stand structure, light, and seedling abundance across a tropical moist Forest chronosequence, Panama. Journal of Vegetation Science, Hoboken, v. 11, n. 2, p. 201-212, 2000.

ENOKI, T.; ABE, A. Saplings distribution in relation to topography and canopy openness in an evergreen broad-leaved Forest. Plant Ecology, New York, v. 173, p. 283-291, 2004.

FERREIRA, L. V.; STOHLGREN, T. J. Effects of river level fluctuation on plant species richness, diversity, and distribution in a floodplain forest in Central Amazonia. Oecologia, Berlin, v. 120, p. 582-587, 1999.

FINEGAN, B. Pattern and process in neotropical secondary rain forest: the first 100 years of sucession. Trends in Ecology \& Evolution, Cambridge, v. 11, p. 119-124, 1996.

FRAZER, G.; CANHAM, C.; LERTZMAN, K. Gap Light Analyzer Version 2: imaging software to extract canopy structure and gap light transmission indices from true-colour fisheye photographs. New York: Simon Fraser University; British Columbia; Institute of Ecosystem Studies, 1999.

GANDOLFI, S. Regimes de luz em florestas estacionais semideciduais e suas possíveis consequências. In: SALES, V. C. C. (Org.). Ecossistemas brasileiros: manejo e conservação. Fortaleza: Expressão, 2003. p. 305-311.

GIEHL, E. L. H.; JARENKOW, J. A. Gradiente estrutural no componente arbóreo e relação com inundações em uma floresta ribeirinha, rio Uruguai, sul do Brasil. Acta Botânica Brasileira, Belo Horizonte, v. 22, n. 3, p. 741-753, 2008.

GILLIAM, F. S.; TURRIL, N. L.; ADAMS, M. B. Herbaceous-layer and overstory species in clear-cut and mature central Appalachian hardwood forest. Ecological application, Washington, v. 5, n. 4, p. 947-955, 1995.

GUARIGUATA, M. R. et al. Structure and floristics of secondary and old-growth forest stand in lowland Costa Rica. Plant Ecology, New York, v. 132, p. 107-120, 1997.

GUARIGUATA, M. R.; OSTERTAG, R. Neotropical secondary forest succession: changes in structural and functional characteristics. Forest Ecology and Management, Amsterdan, v. 148, p. 185-206, 2001.

LEGENDRE, P.; FORTIN, M. J. Spatial pattern and ecological analysis. Vegetatio, New York, v. 80, p. 107-138, 1989.

LYTLE D. A.; POFF, N. L. Adaptation to natural flow regimes. Trends in Ecology \& Evolution, Cambridge, v. 19, n. 2, p. 94-100, 2004.

MAACK, R. Geografia física do Estado do Paraná. Curitiba: Imprensa Oficial do Paraná, 2002.

MARTINI, A. M. Z. Estrutura e composição da vegetação e chuva de sementes em sub-bosque, clareiras naturais e área perturbada por fogo em floresta tropical no sul da Bahia. Tese (Doutorado em Ecologia) - Universidade Estadual de Campinas, Campinas, 2002.

MEIRA-NETO, J. A. A.; MARTINS, F. R. Composição florística do estrato herbáceo-arbustivo de uma floresta estacional semidecidual em Viçosa - MG. Revista árvore, Viçosa, MG, v. 24, n. 4, p. 407-416, 2000.

MONTGOMERY, R. A.; CHAZDON, R. L. Light gradient partitioning by tropical tree seedlings in the absence of canopy gaps. Oecologia, Berlin, v. 131, p. 165-174, 2002.

NEPSTAD, D. C. et al. A comparative study of tree stablishment in abandoned pasture and mature forest of eastern Amazonia. Oikos, Copenhagen, v. 76, p. 25-39, 1996.

NICOTRA, A. B.; CHAZDON, R. L.; IRIARTE, S. Spatial heterogeneity of light and woody seedling regeneration in tropical wet forest. Ecology, Washington, v. 80, p. 1908-1926, 1999.

OLIVEIRA-FILHO, A. T. et al. Effects of soils and topography on the distribution of tree species in a tropical riverine forest in south-eastern Brazil. Journal of Tropical Ecology, Cambridge, v. 10, p. 483-508, 1994.

PEÑA-CLAROS, M. Changes in forest structure and species composition during secondary forest sucession in the bolivian amazon. Biotropica, Lawrence, v. 35, n. 4, p. 450-461, 2003.

PICKETT, S. T. A.; COLLINS, S. L.; ARMESTO, J. J. Models, mechanisms and pathways of sucession. Botanical Review, New York, v. 53, p. 335-371, 1987.

RICHARDS, P. W. The tropical rain forest: an ecological study. 2. ed. Cambridge: Cambridge University 
Press, 1996.

ROSENBERG, M. S. PASSAGE: pattern analysis, spatial statistics, and geographic exegesis. Version 1.1. Tempe: Department of Biology, Arizona State University, 2001.

SCHNITZER, S. A.; DALLING, J. W.; CARSON, W. P. The impact of lianas on tree regeneration in tropical forest canopy gaps: evidence for an alternative pathway of gap-phase regeneration. Journal of ecology, Hoboken, v. 88, p. 655-666, 2000.

SOKAL, R. R.; ROHLF, F. J. Introducción a la bioestatística. Barcelona: Reverté, 1984. (Série de biologia fundamental, n. 5).

SOUZA, M. C.; CISLINSKI, J.; ROMAGNOLO, M. B. Levantamento Florístico. In: VAZZOLER, A. E. A. M.; AGOSTINHO, A. A.; HAHN, N. S. A planície de inundação do Alto Rio Paraná: aspectos físicos, biológicos e socioeconômicos. Maringá: EDUEM, 1997. p. 344-368.

SOUZA, A. F.; MARTINS, F. R. Spatial variation and dynamics of flooding, canopy openness, and structure in a Neotropical swamp forest. Plant Ecology, New York, v. 180, p. 161-173, 2005.

THOMAS, S. M.; ROBERTO, M. C.; BINI L. M. Caracterização limnológica dos ambientes aquáticos e influências dos níveis fluviométricos. In: VAZZOLER, A. E. A. M.; AGOSTINHO, A. A.; HAHN, N. S. A planície de Inundação do Alto Rio Paraná: aspectos físicos, biológicos e socioeconômicos. Maringá: EDUEM, 1997. p. 45-87.

TUOMISTO, H.; POULSEN, A. D. Pteridophyte diversity and species composition in four Amazonian rain forests. Journal of Vegetation Science, Hoboken, v. 11, p. 383-396, 2000.

ZVIEJKOVSKI, I. P. Sucessão em uma pastagem tropical abandonada: mudanças estruturais e estimativas de recuperação florestal. 31 f. 2008. Dissertação (Mestrado em Ecologia) - Universidade Estadual de Maringá, Maringá, 2008. 